\title{
BMJ Open What are the financial implications of an open right hemicolectomy to hospital trusts within NHS England? A cost analysis
}

\author{
Ian Daniels (D) , ${ }^{1}$ Richard Tuson, ${ }^{2}$ Judith Hargreaves ${ }^{3}$
}

To cite: Daniels I, Tuson R, Hargreaves J. What are the financial implications of an open right hemicolectomy to hospital trusts within NHS England? A cost analysis. BMJ Open 2021;11:e053187. doi:10.1136/ bmjopen-2021-053187

- Prepublication history and additional supplemental material for this paper are available online. To view these files, please visit the journal online (http://dx.doi.org/10.1136/ bmjopen-2021-053187).

Received 15 May 2021 Accepted 13 December 2021

D Check for updates

(C) Author(s) (or their employer(s)) 2021. Re-use permitted under CC BY-NC. No commercial re-use. See rights and permissions. Published by BMJ.

${ }^{1}$ Exeter Surgical Health Services Research Unit, Royal Devon and Exeter Hospital, Exeter, UK ${ }^{2}$ Health Analytical Solutions Ltd, Alderley, UK

${ }^{3}$ Medical Solutions Division, 3M Health Care Ltd, Loughborough, UK

Correspondence to

Mr lan Daniels;

i.daniels@nhs.net

\section{ABSTRACT}

Objective This study aimed to quantify the actual costs to National Health Service (NHS) England of open right/ extended right hemicolectomy ( $\mathrm{ORH}$ ) patient episodes compared with national tariffs to determine whether the total cost of care for these patients is adequately reimbursed to NHS Trusts.

Design 2017-2018 NHS Improvement reference cost data for elective and non-elective ORH Healthcare Resource Group 4+ (HRG4+)-coded procedures were used to calculate the actual mean initial admission costs of ORH and compare with corresponding 2017-2018 national tariffs. Costs of postoperative complications were estimated based on 2017-2018 Hospital Episode Statistics (intensive care unit (ICU)/high-dependency unit (HDU) stay and surgical site infection (SSI)) or further associated HRG4+-coded procedures (anastomotic leakage, SSI or hernia).

Setting and patient cohort Data were analysed for all ORH inpatients reported as treated at 140 secondary care Hospital Trusts in England during the 2017-2018 financial year.

Results 9812 ORH HRG4+-coded procedures were reported across 140 Hospital Trusts (74.0\% elective; $26.0 \%$ non-elective). A total 1-year deficit of $£ 993335$ was estimated between actual initial admission costs incurred and tariffs reimbursed for all patient episodes, $93.7 \%$ of which was associated with elective admissions. The cost of the average length of stay (LoS) in ICU/ HDU after an $\mathrm{ORH}$ was $£ 6818$. The additional cost of an extended LoS in ICU/HDU due to an SSI was $£ 45316$. Conclusion The total cost of delivering care for these patients declared by NHS England was far higher than the tariff provided, which may be significantly underestimating the true cost of an $\mathrm{ORH}$, leading to inadequate national tariff-setting by NHS Improvement.

\section{INTRODUCTION}

Colorectal surgery is associated with considerable risks for postoperative complications, which lead to additional costs associated with managing these complications being incurred by the hospital and a significant morbidity burden for the patient. ${ }^{1}$ The most common causes of complications are anastomotic leakage (AL), which can result in further

\section{Strengths and limitations of this study}

- Given the continually rising costs of delivering care within the National Health Service (NHS) in England, this study is timely in its approach to demonstrating cost savings associated with colonic surgery that could be achieved for NHS Trusts.

- As reference cost data is the single largest body of data on costs within the NHS, this study is based on a large enough population to demonstrate a need for improvement.

- As the Healthcare Resource Group code structure allows for increased payments based on complexities and comorbidities, any underlying conditions are factored into the cost matrices, thereby removing associated uncertainty.

- Given the lack of an audit/review of clinical coding and/or finance accounting within NHS England, it may be assumed that some NHS Trust submissions contain errors.

- Due to a lack of data collection and reporting, our analysis cannot demonstrate any costs in primary care; therefore, it is reasonable to conclude that our figures are underestimated.

surgery, ${ }^{23}$ sepsis and the need for intensive care unit (ICU) support and surgical site infection (SSI), ${ }^{3}$ leading to a later surgical site occurrence (SSO), such as an incisional hernia. ${ }^{4}$

The development of a complication may delay discharge from the primary procedure, cause an unplanned readmission following discharge (within 30 days), or even necessitate further surgery to resolve the complication in the long term, such as repair of an incisional hernia or reversal of a stoma. Regardless of the cause, all complications can impact hospital resources and trigger an additional financial burden. ${ }^{5-7}$ In some cases, associated costs can run into tens of thousands of pounds ${ }^{5-7}$; therefore, it is important to ensure that funds received by hospitals 
from those who commission the service adequately cover the costs of treating these patients.

At present, National Health Service (NHS) England does not collect data at a line-item level for all consumables used on an individual patient. Therefore, reference cost data reported on an annual basis to NHS Improvement by individual Trusts are used to inform national tariff setting. Funding between commissioners and healthcare providers within NHS England is managed via Healthcare Resource Groups (HRGs), which determine tariffs for clinically similar treatments that are presumed to use similar levels of healthcare resource. ${ }^{8}$ HRG-coded reference cover the diagnosis, associated treatments, and average length of stay (LoS). This allows patient episodes to be ascribed tariff codes that enable NHS Trusts to charge commissioners accordingly for healthcare services provided. However, this system may be prone to inaccuracies and the true cost of delivering care incurred by healthcare providers may be underestimated.

Our study aimed to highlight the variability in costs associated with a single operation, an open right/extended right hemicolectomy (ORH), which accounted for the majority of colonic surgeries reported within the study period (72\%), and therefore, provided a good sample size, and demonstrate the potential magnitude of the reimbursement shortfall for this patient cohort. Therefore, our objective was to quantify the actual costs to NHS England of initial (index) admissions and postoperative complications resulting in delayed discharge, unplanned readmission or further surgery, associated with ORH patient episodes and compare them with national tariffs.

\section{METHODS}

\section{Patient cohort selection}

A right hemicolectomy (excision of the right colon) and extended right hemicolectomy (excision of the right colon and transverse colon) were selected as suitable examples of open colorectal abdominal surgery for this analysis based on sample size. All Office of Population, Censuses and Surveys fourth revision (OPCS-4.8) Classification of Interventions and Procedures codes relevant to the right hemicolon or transverse colon were then identified based on published lists ${ }^{9}$ and confirmed via expert clinical opinion (OPCS-4.8 H06+, H07 +and H08+; online supplemental table S1). To quantify actual costs of initial (index) admission patient episodes and associated tariffs, identified OPCS-4.8 codes were subsequently mapped to HRG4 +codes FF32A, FF32B and FF32C (Proximal colon procedures; 19 years and over; and with complexity and comorbidity (CC) score 6+, CC score 3-5, or CC score 0-2, respectively) based on published lists ${ }^{10}$ and confirmed via expert clinical opinion.

\section{Estimation of costs of initial (index) admission ORH patient episodes}

The 2017-2018 NHS Improvement reference cost data for HRG4 +s FF32A, FF32B and FF32C were used to calculate the actual initial admission cost of ORH patient episodes incurred by healthcare providers. ${ }^{11}$ Reference cost data for a small number of outliers undergoing colonic surgery as outpatients were excluded from this analysis and assumed to be inaccuracies of coding, since open surgery requires an inpatient stay. These figures were then compared with 2017-2018 national tariffs published by NHS Improvement. ${ }^{12}$ Both reference cost and national tariff data used in this comparison were categorised as elective (ie, planned) or non-elective (ie, emergency) admissions to identify variations in cost and reimbursement.

A further subanalysis considered only those patients with greater complexity and/or more comorbidities (ie, HRG4 +FF32A-coded procedures) to explore the possible association between case mix and actual initial admission costs reported by individual Trusts for these patient episodes.

\section{Estimation of costs of postoperative complications resulting} in delayed discharge of patients who underwent ORH

In a separate analysis, Hospital Episode Statistics (HES) ${ }^{13}$ were analysed to better understand the actual costs associated with postoperative complications that may delay discharge from a primary ORH procedure, such as SSIs and ICU or high-dependency unit (HDU). The 2017-2018 HES data were used to quantify the number of patients who underwent an ORH based on the OPCS-4.8 codes identified (as described above, online supplemental table S1) and categorised as elective or nonelective admissions. OPCS-4.8 code Y752 (Laparoscopic approach to abdominal cavity not elsewhere classified) was used to exclude patients who did not undergo an ORH from the analysis. To demonstrate the variability in treatment required across this patient cohort, it was relevant to include patients with and without complications in this analysis. Therefore, mean surgical ward LoS was reported for all patient episodes, as well as the proportion of patients who were admitted to a critical care facility (ICU/HDU) or had an SSI (identified by International Classification of Diseases 10th Revision diagnostic code T814), and the respective impact of an ICU/HDU stay, SSI or both, on LoS. The mean increase in surgical ward stay cost for patients with an SSI was also calculated based on the NHS Improvement 2017-2018 bed day tariff ${ }^{11}$ and previously described HES LoS data. ${ }^{13}$ 2017-2018 NHS England reference cost data for a single ICU/HDU day (Critical care HRG4 +codes XC01Z-XC07Z) ${ }^{11}$ were then used in combination with HES LoS data (as described above $)^{13}$ to estimate the mean cost of ICU/HDU stays for these patients, with and without an SSI.

Estimation of costs of postoperative complications resulting in unplanned readmission or further surgery in patients who underwent $\mathrm{ORH}$

Further HRG4+-coded procedure reference costs were also identified, based on published lists ${ }^{10}$ and expert clinical opinion, and analysed to better understand the actual 
costs associated with the development of common postoperative complications requiring readmission (eg, AL or SSI), sometimes leading to SSOs (eg, incisional hernia) in patients initially admitted for ORH procedures.

The actual cost of patient readmissions due to postoperative complications was estimated for the most and least complex and/or comorbid cases using reference cost data for HRG4 +codes FF51A and FF51E (Major general abdominal procedures; 19 years and over; and with CC score $10+$, or CC score 0 , respectively), ${ }^{7}$ and compared with national tariff data. ${ }^{12}$ Similarly, the actual cost of patients representing with complications requiring additional surgery was estimated for the most and least complex and/or comorbid cases using reference cost data for HRG4 + codes FF31A and FF31D (Complex large intestine procedures; 19 years and over; and with CC score $9+$, or CC score $0-2$, respectively), ${ }^{11}$ and compared with national tariff data. ${ }^{12}$ All data used in these comparisons were categorised as non-elective admissions, that is, emergency admissions.

Finally, the actual cost of patients subsequently representing with an incisional hernia was estimated for the least complex and/or comorbid cases using reference cost data for HRG4 +code FF61C (Abdominal hernia procedures; 19 years and over; and with CC score 0), ${ }^{11}$ and compared with national tariff data. ${ }^{12}$ All data used in this comparison were categorised as elective or nonelective admissions to identify variations in cost and reimbursement. A summary table of all data sources and uses is included in online supplemental table S2.

\section{NHS England Trusts}

To assess the financial burden of patients who underwent an ORH at a national level, information from all NHS
England Trusts that submitted both reference cost data to NHS Improvement and HES data reporting activity levels for the 2017-2018 financial year were included in this analysis.

\section{Statistical methods}

All statistical analyses were carried out using Microsoft Excel (Microsoft, 2016 version for Windows). For parametric HRG4 +data, mean \pm SD and range were reported. For HES data, descriptive statistics were reported (mean for continuous variables and number (percentage) for categorical variables).

\section{Patient and public involvement}

No patient involved.

\section{RESULTS}

One-hundred and forty of a possible 150 NHS England Trusts (93.3\%) submitted both reference cost data to NHS Improvement for HRG4 +FF32 and HES data reporting activity levels for these procedures for the 2017-2018 financial year. In total, 9812 HRG4 +FF32 codes were reported between 1 April 2017 and 31 March 2018 across all 140 NHS England Trusts (table 1, figure 1). Elective admissions (ie, planned admissions) accounted for $74.0 \%$ (7260/9812) HRG4 +FF32 codes reported while non-elective admissions (ie, emergency admissions) accounted for $26.0 \%$ (2552/9812). Overall, ORH procedures in patients $\geq 19$ years with lower complexity and/or fewer comorbidities (HRG4 +FF32C-coded procedures) accounted for $65.3 \%$ of reported activity across all Trusts $(6,408 / 9,812)$. There were more elective admissions than non-elective admissions across all HRG4 +FF32 codes.

Table 1 Total Healthcare Resource Group 4 (HRG)+FF32 codes reported in 2017-2018 financial year by 140 National Health Service England Trusts, total actual initial (index) admission costs (2017-2018) and corresponding national tariff totals (20172018), categorised by code and by elective versus non-elective admission

\begin{tabular}{|llll}
\hline HRG4 +code & $\begin{array}{l}\text { Sum of actual initial (index) } \\
\text { admission costs reported }\end{array}$ & Sum of national tariffs & $\begin{array}{l}\text { Difference between } \\
\text { tariff and actual initial } \\
\text { (index) admission costs }\end{array}$ \\
\hline FF32A-all $(\mathrm{N}=1020)$ & $£ 9446648$ & $£ 9221238$ & $-£ 225410$ \\
Elective $(n=582)$ & $£ 5369086$ & $£ 7945 \times 582=£ 4623990$ & $-£ 745096$ \\
\hline Non-elective $(n=438)$ & $£ 4077562$ & $£ 10496 \times 438=£ 4597248$ & $£ 519686$ \\
\hline FF32B-all $(\mathrm{N}=2384)$ & $£ 16990583$ & $£ 15223688$ & $-£ 1766895$ \\
\hline Elective $(n=1694)$ & $£ 11910822$ & $£ 5947 \times 1694=£ 10074218$ & $-£ 1836604$ \\
\hline Non-elective $(n=690)$ & $£ 5079761$ & $£ 7463 \times 690=£ 5149470$ & $£ 69709$ \\
\hline FF32C-all $(\mathrm{N}=6408)$ & $£ 41083106$ & $£ 33142176$ & $-£ 7940930$ \\
\hline Elective $(n=4984)$ & $£ 31915371$ & $£ 5056 \times 4984=£ 25199104$ & $-£ 6716267$ \\
\hline Non-elective $(n=1424)$ & $£ 9167735$ & $£ 5578 \times 1424=£ 7943072$ & $-£ 1224663$ \\
\hline Total FF32-all $(N=9812)$ & $£ 67520337$ & $£ 57587102$ & $-£ 9933235$ \\
\hline Elective $(n=7260)$ & $£ 49195279$ & $£ 39897312$ & $-£ 9297967$ \\
Non-elective $(n=2552)$ & $£ 18325058$ & $£ 17689790$ & $-£ 635268$ \\
\hline
\end{tabular}

Elective, planned; non-elective, emergency. 


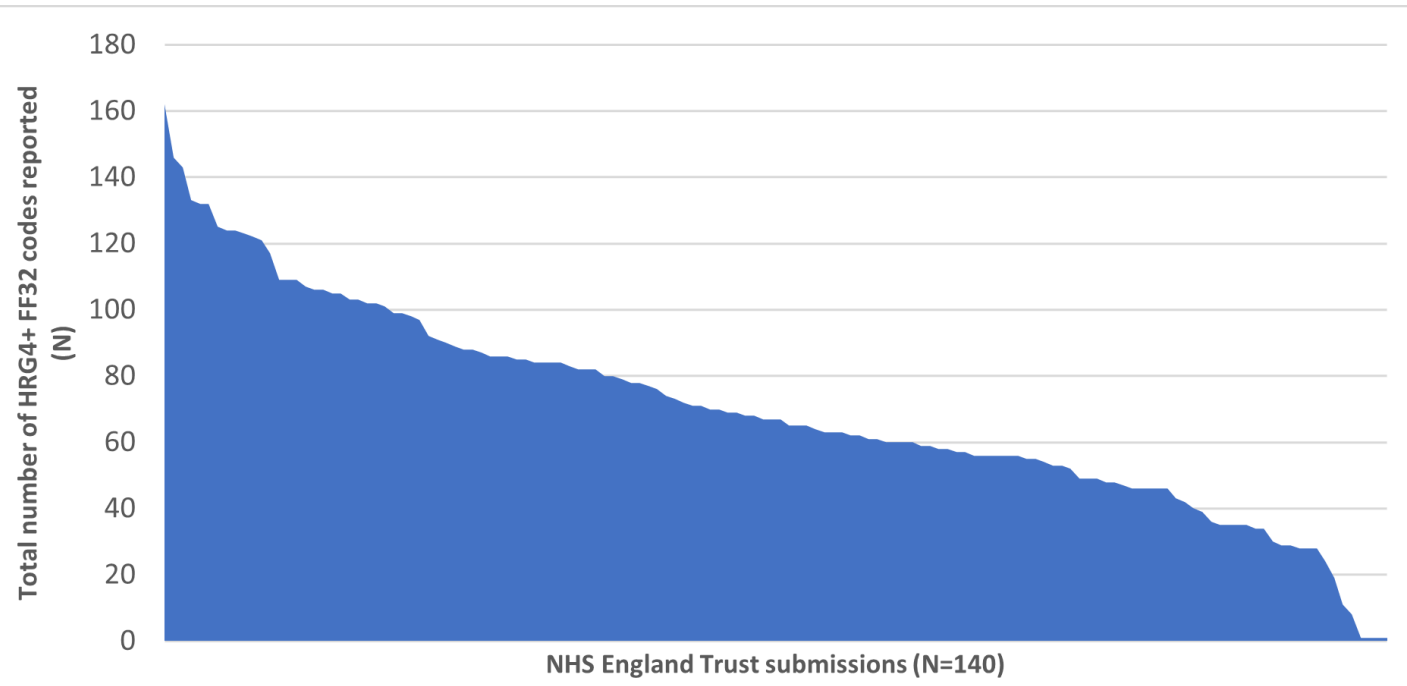

Figure 1 Total number of Healthcare Resource Group 4 (HRG)+FF32 codes reported in 2017-2018 financial year by each National Health Service (NHS) England Trust.

\section{Costs of initial (index) admission ORH patient episodes}

The total actual costs of initial admissions for ORH procedures (HRG4 +s FF32A, FF32B and FF32C) incurred by 140 NHS England Trusts during the 2017-2018 financial year (categorised as elective or non-elective admissions) are shown in table 1 , alongside the corresponding HRG4 +2017-2018 national tariff totals for comparison. These figures suggest a total 1-year deficit between actual initial admission patient episode costs incurred and tariffs reimbursed across all 140 NHS England Trusts of $£ 9933235$. Of this deficit, $93.6 \%$ was associated with elective admissions.

For all elective and non-elective HRG4 +FF32-coded procedures included in this analysis, mean $\pm \mathrm{SD}$ actual initial admission costs per patient episode of $£ 7439 \pm$ $£ 3167$ and $£ 7093 \pm £ 4095$, respectively, were reported in 2017-2018 (table 2). The weighted average national tariffs for these procedures during 2017-2018 were $£ 5496$ and £6932, respectively, suggesting a potential loss of $£ 1944$ per elective patient procedure and a smaller potential loss of $£ 162$ per non-elective patient procedure for healthcare providers. Reference costs, reported by providers of these procedures, for all elective and nonelective HRG4 +FF32-coded procedures included in this analysis showed considerable variation, ranging from $£ 83$ to $£ 25722$ and from $£ 20$ to $£ 30355$, respectively.

\section{Subanalysis on initial (index) admission costs of most complex patient episodes}

The most complex patient episodes (ie, HRG4 +FF32Acoded procedures), reported by 133 of 140 Trusts, accounted for $8.0 \%(582 / 7260)$ of all elective procedures and $17.2 \%(438 / 2552)$ of all non-elective procedures analysed across all Trusts (table 1). Reported reference costs for these procedures ranged from £230 to £25 722 and from $£ 113$ to $£ 30$ 355, respectively, suggesting surprisingly low costs reported for complex patients in some cases, which are presumed inaccuracies in coding. However, mean \pm SD actual initial admissions costs per patient episode of $£ 9182 \pm £ 3871$ and $£ 8933 \pm £ 5353$, respectively, were reported (table 2, figures 2 and 3 ). Given the 2017-2018 HRG4 +FF32A national tariffs for elective and non-elective admissions, these results suggest an average loss of $£ 1237$ per complex elective patient procedure but more than adequate reimbursement for healthcare providers per complex non-elective patient procedure (table 2 ).

Across all NHS England Trusts, $45.3 \%$ of the total reported HRG4 +FF32A elective admission activity was reported as costing above the calculated mean actual initial admission cost of $£ 9182$ (figure 2). Furthermore, $12.9 \%$ of the total reported HRG4 +FF32A elective admission activity cost was $>1 \mathrm{SD}(>£ 3871)$ above the calculated mean actual initial admission cost. This cohort of $12.9 \%$ of patients had an elevated mean actual initial admission cost per patient episode of $£ 15974$.

A parallel analysis on HRG4 +FF32A non-elective admissions yielded similar results. Across all NHS England Trusts, $49.3 \%$ of the total reported HRG4 +FF32A nonelective admission activity was reported as costing above the calculated mean actual initial admission cost of $£ 8933$ (figure 3). Similarly, $12.6 \%$ of the total reported HRG4 +FF32A non-elective admission activity cost was $>1 \mathrm{SD}(>£ 5,353)$ above the calculated mean actual initial admission cost. This cohort of $12.6 \%$ of patient had a mean actual initial admission cost per patient episode of $£ 18717$.

\section{Costs of postoperative complications resulting in delayed} discharge of patients who underwent ORH

Surgical ward stays, ICU/HDU stays and SSIs

Between 1 April 2017 and 31 March 2018, HES for patients who underwent an ORH (ie, OPCS-4.8-coded $\mathrm{H} 06+, \mathrm{H} 07$ +and $\mathrm{H} 08+$ procedures) reported across all 140 
Table 2 Mean \pm SD and range of actual costs for all analysed Healthcare Resource Group 4 (HRG)+-coded patient episodes reported by 140 National Health Service England Trusts (2017-2018) and corresponding weighted average national tariffs (2017-2018), categorised as elective or non-elective admissions (where appropriate)

\begin{tabular}{|c|c|c|c|c|}
\hline HRG4 +code & Mean \pm SD actual cost reported & $\begin{array}{l}\text { Range of actual costs } \\
\text { reported }\end{array}$ & $\begin{array}{l}\text { Weighted average } \\
\text { national tariff }\end{array}$ & $\begin{array}{l}\text { Difference } \\
\text { between tariff and } \\
\text { actual cost }\end{array}$ \\
\hline \multicolumn{5}{|c|}{ Initial (index) admission costs } \\
\hline \multicolumn{5}{|c|}{ FF32-all } \\
\hline Elective & $£ 7439 \pm £ 3167$ & $£ 83-£ 25722$ & $£ 5496$ & $-£ 1944$ \\
\hline Non-elective & $£ 7093 \pm £ 4095$ & $£ 20-£ 30355$ & $£ 6932$ & $-£ 162$ \\
\hline \multicolumn{5}{|l|}{ FF32A } \\
\hline Elective & $£ 918 \pm £ 3871$ & $£ 230-£ 25722$ & $£ 7945$ & $-£ 1237$ \\
\hline Non-elective & $£ 8933 \pm £ 5353$ & $£ 113-£ 30355$ & $£ 10496$ & $+£ 1563$ \\
\hline \multicolumn{5}{|l|}{ FF32B } \\
\hline Elective & $£ 7281 \pm £ 2734$ & $£ 509-£ 23935$ & $£ 5947$ & $-£ 1784$ \\
\hline Non-elective & $£ 6964 \pm £ 3511$ & $£ 20-£ 19592$ & $£ 7463$ & $+£ 499$ \\
\hline \multicolumn{5}{|l|}{ FF32C } \\
\hline Elective & $£ 6405 \pm £ 2405$ & $£ 83-£ 17723$ & $£ 5056$ & $-£ 1349$ \\
\hline Non-elective & $£ 6011 \pm £ 3041$ & $£ 73-£ 21585$ & $£ 5578$ & $-£ 433$ \\
\hline \multicolumn{5}{|c|}{ Costs of postoperative complications resulting in unplanned readmission or further surgery } \\
\hline \multicolumn{5}{|c|}{ FF51A } \\
\hline Elective & N/A & N/A & N/A & N/A \\
\hline Non-elective & $£ 8,985 \pm £ 9017$ & $£ 61-£ 89389$ & $£ 11541$ & $+£ 2556$ \\
\hline \multicolumn{5}{|l|}{ FF51E } \\
\hline Elective & N/A & N/A & N/A & N/A \\
\hline Non-elective & $£ 3664 \pm £ 3092$ & $£ 99-£ 50646$ & $£ 2935$ & $-£ 729$ \\
\hline \multicolumn{5}{|l|}{ FF31A } \\
\hline Elective & N/A & $\mathrm{N} / \mathrm{A}$ & N/A & $\mathrm{N} / \mathrm{A}$ \\
\hline Non-elective & $£ 11048 \pm £ 10066$ & $£ 20-£ 87003$ & $£ 12347$ & $+£ 1299$ \\
\hline \multicolumn{5}{|l|}{ FF31D } \\
\hline Elective & N/A & N/A & N/A & N/A \\
\hline Non-elective & $£ 6630 \pm £ 5753$ & $£ 20-£ 109516$ & $£ 6520$ & $-£ 110$ \\
\hline \multicolumn{5}{|l|}{ FF61C } \\
\hline Elective & $£ 2862 \pm £ 1800$ & £8-£16833 & $£ 1872$ & $-£ 990$ \\
\hline Non-elective & $£ 2974 \pm £ 1628$ & $£ 242-£ 12147$ & $£ 2650$ & $-£ 324$ \\
\hline
\end{tabular}

Elective, planned; N/A, not applicable; non-elective, emergency.

NHS England Trusts $(\mathrm{N}=6667)$ indicated a mean surgical ward LoS for all patient episodes of 13.3 days per patient (table 3). Mean surgical ward LoS for patients with an SSI increased to 29.0 days per patient, an additional 15.7 days per patient above the mean for all ORH patient episodes. The cost of the average extended surgical ward LoS associated with an SSI was estimated at £6139 using the published 2017-2018 NHS England surgical ward bed day rate of $£ 391 .{ }^{12}$

Furthermore, $2.0 \%(133 / 6,667)$ of patients who underwent an ORH (including elective and non-elective procedures) were admitted to an ICU/HDU for a mean LoS of 6.5 days per patient. Of the patients requiring ICU/ HDUs, $18.8 \%$ had an SSI (25/133), which increased the mean LoS in ICU/HDU to 49.7 days per patient, an additional 43.2 days per patient above the mean for all ORH patient episodes. NHS England reference costs for a single ICU/HDU day are stratified by number of organs supported (online supplemental table S3) and 20172018 actual reported costs ranged from $£ 693$ per day for zero organs supported to $£ 2359$ per day for six or more organs supported, with a weighted average cost of $£ 1395$. However, HRG4 + code XC06Z (Adult critical care, 1 organ supported, £1049 per day) was considered the most likely HRG4 +code to be used for most patients with an ORH in ICU/HDU. Therefore, based on a cost of $£ 1049 /$ day, the cost of the average LoS in ICU/HDU associated with a patient transferred to ICU/HDU after an ORH was estimated at $£ 6818$ and the additional cost of an extended LoS in ICU/HDU as a result of an SSI was estimated at $£ 45316$. 


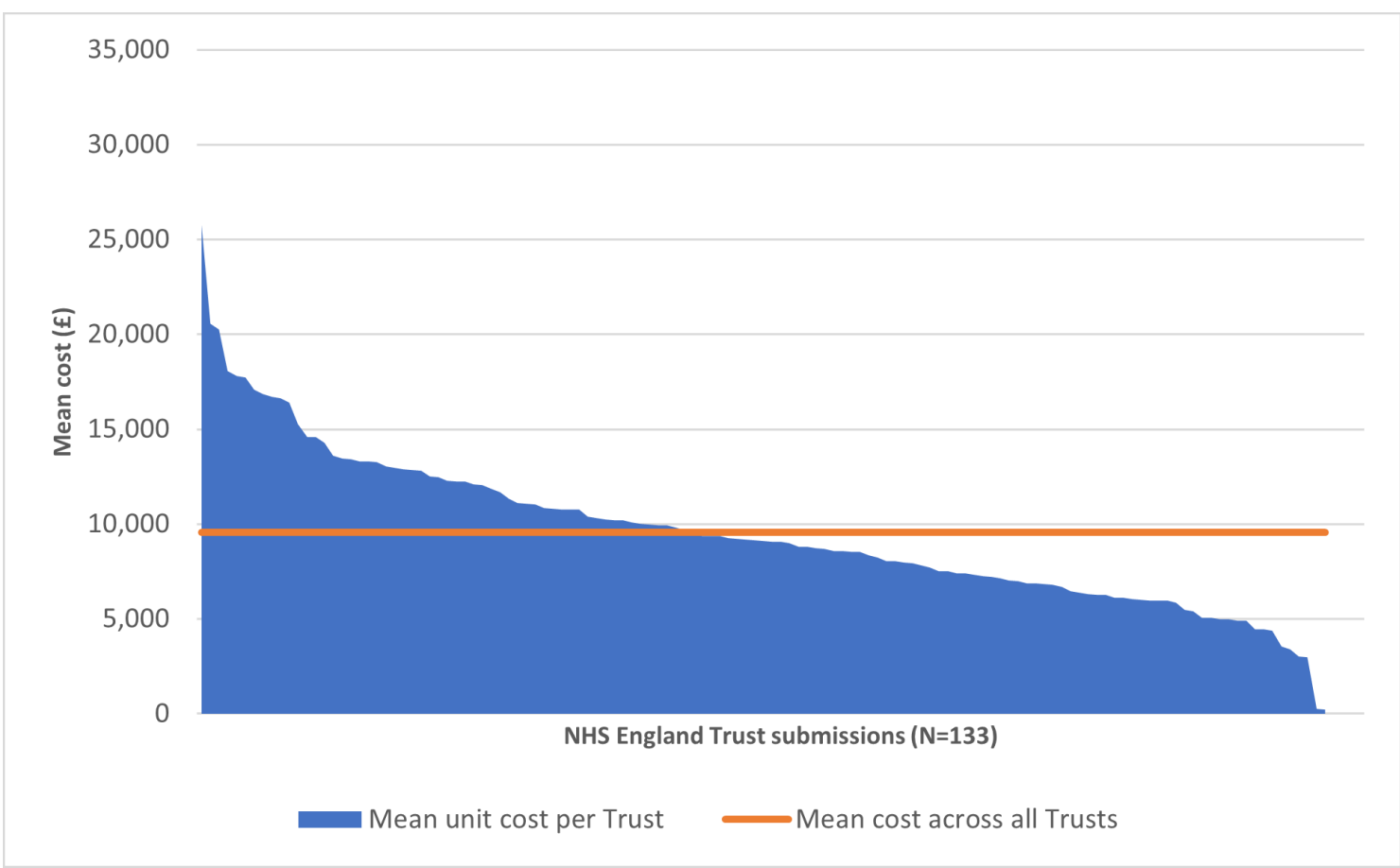

Figure 2 Range of actual initial (index) admission patient episode costs of Healthcare Resource Group 4+FF32A-coded procedures incurred by 133 NHS England Trusts (2017-2018) for elective admissions (ie, planned). NHS, National Health Service.

Costs of postoperative complications resulting in unplanned readmission or further surgery in patients who underwent $\mathrm{ORH}$

Unplanned patient readmissions

Reported 2017-2018 reference costs for non-elective patient readmissions assigned HRG4 +FF51A (Major general abdominal procedures, 19 years and over, with CC score 10+) and FF51E (Major general abdominal procedures, 19 years and over, with CC score 0 ) codes (table 2), also showed significant ranges: from $£ 61$ to $£ 89389$ and from $£ 99$ to $£ 50$ 646, respectively. Compared with weighted average tariffs for patient episodes associated

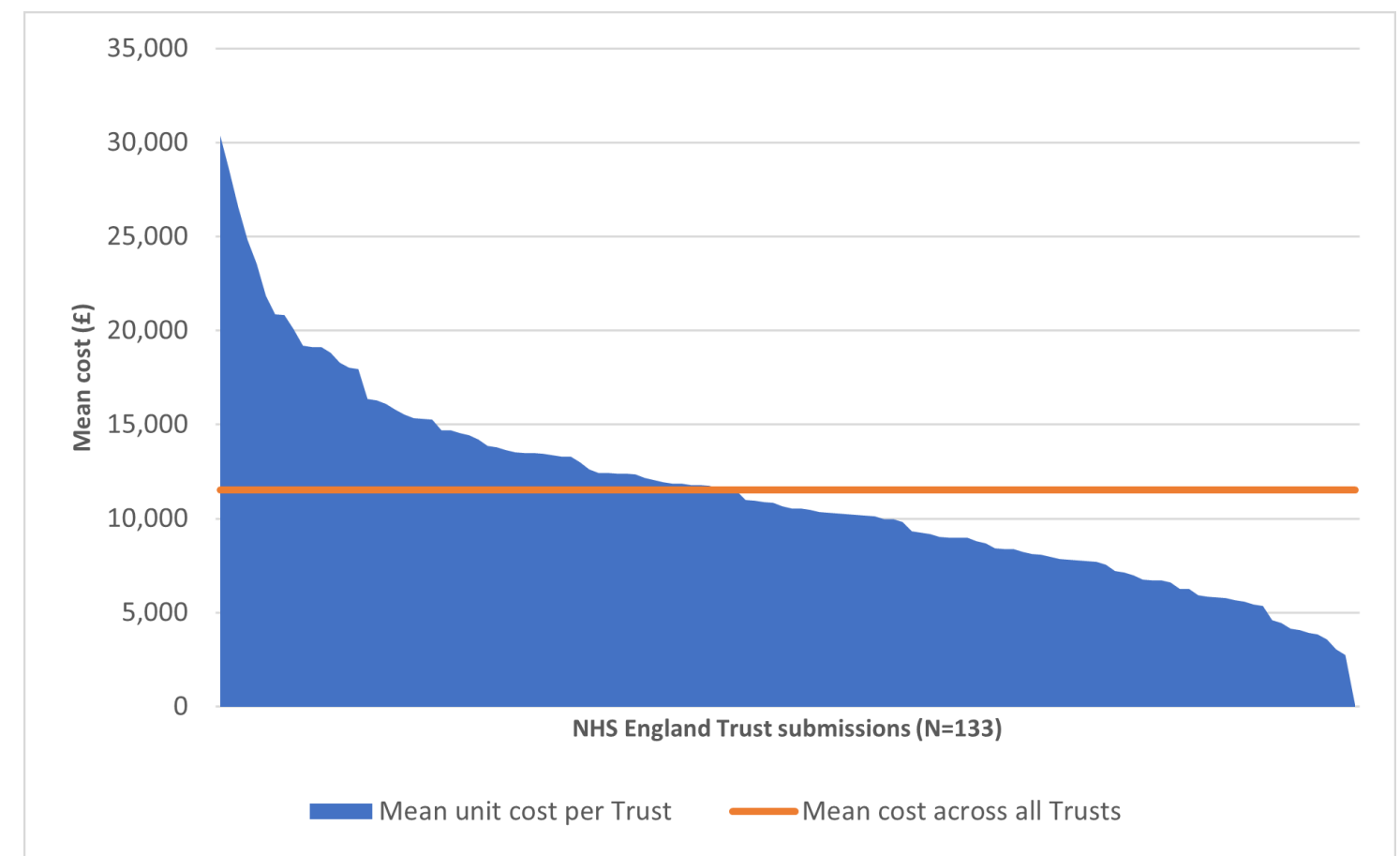

Figure 3 Range of actual initial (index) admission patient episode costs of Healthcare Resource Group 4+FF32A-coded procedures incurred by 133 NHS England Trusts (2017-2018) for non-elective admissions (ie, emergency). NHS, National Health Service. 
Table 3 Hospital Episode Statistics for patients with an open right or extended right hemicolectomy reported in 2017-2018 financial year by 140 National Health Service England Trusts ( $\mathrm{N}=6667)$

Surgical ward LoS, all patient episodes, 13.3 days/patient mean

$\begin{array}{ll}\text { No of patients with SSI, n (\%) } & 364(5.5 \%) \\ \begin{array}{l}\text { Surgical ward LoS, patients with SSI, } \\ \text { mean }\end{array} & 29.0 \text { days/patient }\end{array}$

\begin{tabular}{|c|c|}
\hline No of patients with ICU/HDU stay, n (\%) & $133(2.0 \%)$ \\
\hline $\begin{array}{l}\text { LoS in ICU/HDU, patients with ICU/HDU } \\
\text { stay, mean }\end{array}$ & 6.5 days/patient \\
\hline $\begin{array}{l}\text { No of patients with ICU/HDU stay and } \\
\text { SSI, n (\%) }\end{array}$ & $25(18.8 \%)^{*}$ \\
\hline $\begin{array}{l}\text { LoS in ICU/HDU, patients with ICU/HDU } \\
\text { stay and SSI, mean }\end{array}$ & 49.7 days/patient \\
\hline
\end{tabular}

${ }^{*}$ Percentage calculated as proportion of patients with ICU stay. HDU, high-dependency unit; ICU, intensive care unit; LoS, length of stay; SSI, surgical site infection.

with these procedures, mean $\pm \mathrm{SD}$ actual costs suggested more than adequate reimbursement for more complex non-elective FF51A procedures but a potential loss of $£ 729$ per less complex non-elective FF51E procedure for healthcare providers.

\section{Patients representing requiring additional colorectal surgery}

Reported 2017-2018 reference costs for patients requiring additional non-elective colorectal surgery assigned HRG 4+FF31A (Complex large intestine procedures, 19 years and over, with CC score 9+) and FF31D (Complex large intestine procedures, 19 years and over, with CC score 0-2) codes (table 2), also showed significant ranges from $£ 20$ to $£ 87003$ and $£ 20$ to $£ 109516$, respectively. Similarly, mean $\pm \mathrm{SD}$ actual costs suggested more than adequate reimbursement for more patient episodes associated with complex non-elective FF31A additional colorectal surgery but a potential loss of $£ 110$ per less complex non-elective FF51E additional colorectal surgery patient episode, when compared with weighted average tariffs for these procedures.

\section{Patients representing with an incisional hernia}

When considering only the least complex patients representing with an incisional hernia (ie, HRG4 +FF61Ccoded procedures), despite smaller ranges in reference costs reported for both elective and non-elective admissions, the results suggest average losses for healthcare providers of $£ 990$ per elective incisional hernia patient episode and $£ 324$ per non-elective incisional hernia patient episode (table 2).

\section{DISCUSSION}

Our study demonstrates the significant financial burden of one subset of colorectal surgical procedures (ie, ORH) to NHS England. We estimated a total 1-year deficit of almost $£ 10000000.00$ between actual initial (index) admission costs incurred and tariffs reimbursed for almost 10000 ORH procedures across 140 NHS England Trusts, even before factoring in the costs of postoperative complications associated with these patient episodes. At a top line level, this deficit illustrates the 'out of kilter' setting of tariffs versus the true costs to NHS Trusts; primary open surgery is shown to be more costly than the funding hospitals receive when tariffs are calculated based on cost means. Further analyses of the data demonstrated the variability in patient episodes coded similarly and explored the impact on costs.

The true impact of underfunded tariffs is masked as the range of reference costs reported by NHS Trusts for a procedure, which is used by NHS Improvement to set the tariff, is extensive. Three-quarters of the procedures in this analysis were elective admissions, accounting for more than $90 \%$ of the estimated deficit, and the range of costs submitted by NHS Trusts for elective ORH initial admissions was considerable (from about £80-£26000 per patient episode). Perhaps understandably, the range of costs for unscheduled non-elective ORH initial admissions was greater (from about $£ 20-£ 30000$ per patient episode), although these costs only accounted for a small proportion of the total estimated deficit. Furthermore, when considering the most complex patient episodes, which accounted for more non-elective than elective procedures, $49.3 \%$ and $45.3 \%$ of reported activity, respectively, was above the calculated mean actual initial admission cost for these procedures, with $12.6 \%$ and $12.9 \%$, respectively, $>1 \mathrm{SD}$ above the mean. The results of this analysis suggest that outliers impacted the mean actual initial admission cost for both elective and non-elective procedures, as evidenced by the total 1-year deficit. Although there were a significant number of high-cost episodes reported, the effect of the very low-cost episodes reported appeared to impact the tariff to a greater extent, thereby reducing payments for complex patients. Based on these findings, we suggest that cost control for this type of surgery could benefit from a review, with consideration given to when and where the surgery should take place and assessed against evidence-based practice as outlined by the NHS improvement programme Getting It Right First Time. ${ }^{14}$ Given that the majority of NHS Trusts are performing ORH procedures throughout England, with reported costs ranging from $£ 20$ to over $£ 30000$, it is our view that complex patients should potentially be centralised.

We also assessed the costs of common postoperative complications resulting in delayed discharge, unplanned readmission or further surgery associated with ORH. Our findings showed that the SSI rate for these patients and the impact on bed days have significant consequences for NHS Trusts. The National Institute for Health and Care Excellence (NICE) reports the average cost of a large bowel SSI in the NHS at $£ 5518$. ${ }^{15}$ We demonstrated that an SSI increased the average surgical ward LoS by 15.7 bed days at an additional cost of over $£ 6000$ per patient. 
This $118 \%$ increase in bed days uses vital bed capacity, which could be a factor with respect to recent reporting of waiting times within NHS England Trusts. ${ }^{16}$ Moreover, this study only considered ORH patient episodes. We did not consider laparoscopic right hemicolectomies, which are reported to have a higher cost despite a reported shorter LoS. ${ }^{17}$ It must also be recognised that there are many open (and laparoscopic) colorectal procedures that could potentially have an equivalent, if not greater, impact on bed capacity.

Some patients do require ICU/HCU care. However, with the risk of an SSI because of an ORH, it was reported that each patient who presented with an SSI leading to an ICU/HCU admission cost more than an additional $£ 45000$ due to an average extended LoS of 43.2 days (49.7-6.5 days). Moreover, this calculation was based on the patient having a single organ supported, whereas in some cases, the number of organs supported could be greater, significantly increasing the associated costs. This led to a $665 \%$ increase in mean LoS in ICU/HDU due to an SSI. Once again, this impacts on already overstretched resources.

With a number of patient pathways, it has been extensively reported that readmissions to hospital are common due to later events, such as incisional hernia development. ${ }^{18}$ We found that the corresponding national tariffs for the most complex non-elective patient readmissions or representations requiring additional surgery were more than adequate, likely because the initial presentation was elective and the later readmission was often for a non-elective procedure and, therefore, funded separately. However, national tariffs for less complex readmissions or representations fell short of mean reported costs. Similarly, national tariffs for the least complex patients representing with incisional hernias, whether elective or non-elective, were also insufficient to cover healthcare providers' costs.

\section{Limitations}

This study has identified several limitations with respect to the reference cost data due to current data collection and reporting processes within and across NHS Trusts. The data used for this analysis was of higher quality than that available in previous years, as 2017-2018 reference cost data includes NHS Trust-submitted patient-level information costing system. These are reported at a more granular level, for the first time, and should, therefore, more accurately reflect the true cost of patient care. However, the quality of NHS Improvement reference cost data is not audited; therefore, we were unable to remove any outliers. In this regard, the range of actual costs reported and the SDs associated with mean actual costs reported throughout this study provide further evidence of varied cost collection and submission procedures for complex surgeries across NHS England. This could impact the overall mean costs reported; however, we have shown a weighted average in all cases where data were available.
Unfortunately, our analysis was unable to demonstrate any costs in primary care, due to the lack of relevant data collection and reporting in this setting. Therefore, it is reasonable to suggest that our findings are an underestimate of the total cost of patient care.

Furthermore, HES for complex surgeries are reliant on the clinical codes assigned by hospital coding teams, which are translated from the surgeons' operation notes. The latest NHS England report indicated a mean margin of error of $7.0 \%$ (range $1.1 \%-45.8 \%$ ) in clinical coding of HES data across the country. ${ }^{19}$ Although these errors influence the HRG4 +groupings, they do not change the actual costs associated with delivering surgeries. As NHS England evolves towards reporting more patient-level cost data for all treatments, greater clarity should emerge. It is highly likely that this will generate a greater focus on actual cost submissions, challenging NHS Trusts on irregular cost reporting.

\section{CONCLUSION}

After completing the analysis of both the initial (index) admission costs, tariffs and financial implications of postoperative complications, it is clear the total costs of delivering care for patients who undergo an ORH declared by all NHS Trusts is far higher than the tariff provided. In addition to underfunded initial admission cost tariffs for some patient episodes, postoperative complication costs add to the total cost of care for many patients but are not always adequately reimbursed to NHS Trusts.

These findings demonstrate a potential lack of understanding of the costing system, evidenced by NHS Trusts submitting reference costs as low as $£ 20$ for a non-elective primary ORH procedure seemingly without thought to the impact on the national tariff, and a need to better understand the optimum pathway for these patients. Significant savings could potentially be achieved by better recognition of the range of costs contributing to the complete care of these patients.

Although some patients will require a planned elective or postoperative ICU/HDU stay, aftercare is crucial to ensure avoidable readmission costs are either reduced or eliminated where possible. By using evidence-based care and comparing this to individual patient pathways, it may be possible to reduce SSIs and other complications in the future, thereby potentially eliminating the cost of managing these complications. System changes such as these would benefit both NHS England and patients.

Twitter Richard Tuson @hasdata

Acknowledgements The authors would like to acknowledge Sue Williamson and Caroline Spencer (Rx Communications, Mold, UK) for medical writing assistance with the preparation of this manuscript, funded by $3 \mathrm{M}$ Health Care, Medical Solutions Division.

Contributors ID: conception of the work, interpretation of data for the work, critical revision of the manuscript for important intellectual content. JH: conception of the work, design of the work, interpretation of data for the work, critical revision of the manuscript for important intellectual content. RT: acquisition of data for the work, 
analysis of data for the work, interpretation of data for the work, drafting of the manuscript. ID: gaurantor of the work.

Funding This manuscript was funded by 3M Health Care, Medical Solutions Division, UK. Award/Grant number is not applicable.

Competing interests ID has received honoraria for research, lecturing and acting as an advisor to a number of companies although has no conflict of interest related directly to this work. $\mathrm{JH}$ is an employee of $3 \mathrm{M}$ Health Care, Medical Solutions Division, UK. Health Analytical Solutions (RT) provides health-economic services to 3M.

Patient consent for publication Not applicable.

Ethics approval This study did not require ethical approval as all data used were already in the public domain.

Provenance and peer review Not commissioned; externally peer reviewed.

Data availability statement Data are available in a public, open access repository.

Supplemental material This content has been supplied by the author(s). It has not been vetted by BMJ Publishing Group Limited (BMJ) and may not have been peer-reviewed. Any opinions or recommendations discussed are solely those of the author(s) and are not endorsed by BMJ. BMJ disclaims all liability and responsibility arising from any reliance placed on the content. Where the content includes any translated material, BMJ does not warrant the accuracy and reliability of the translations (including but not limited to local regulations, clinical guidelines, terminology, drug names and drug dosages), and is not responsible for any error and/or omissions arising from translation and adaptation or otherwise.

Open access This is an open access article distributed in accordance with the Creative Commons Attribution Non Commercial (CC BY-NC 4.0) license, which permits others to distribute, remix, adapt, build upon this work non-commercially, and license their derivative works on different terms, provided the original work is properly cited, appropriate credit is given, any changes made indicated, and the use is non-commercial. See: http://creativecommons.org/licenses/by-nc/4.0/.

ORCID iD

Ian Daniels http://orcid.org/0000-0002-9114-0812

\section{REFERENCES}

1 Scarborough JE, Schumacher J, Kent KC, et al. Associations of specific postoperative complications with outcomes after elective colon resection: a procedure-targeted approach toward surgical quality improvement. JAMA Surg 2017;152:e164681.

2 Yoshida T, Miyata $\mathrm{H}$, Konno $\mathrm{H}$, et al. Risk assessment of morbidities after right hemicolectomy based on the National clinical database in Japan. Ann Gastroenterol Surg 2018;2:220-30.

3 Kirchhoff P, Clavien P-A, Hahnloser D. Complications in colorectal surgery: risk factors and preventive strategies. Patient Saf Surg 2010;4:5.
4 Al Chalabi H, Larkin J, Mehigan B, et al. A systematic review of laparoscopic versus open abdominal incisional hernia repair, with meta-analysis of randomized controlled trials. Int J Surg 2015;20:65-74.

5 Healy MA, Mullard AJ, Campbell DA, et al. Hospital and payer costs associated with surgical complications. JAMA Surg 2016;151:823-30.

6 Straatman J, Cuesta MA, de Lange-de Klerk ESM, et al. Hospital cost-analysis of complications after major abdominal surgery. Dig Surg 2015;32:150-6.

7 Vonlanthen R, Slankamenac K, Breitenstein S, et al. The impact of complications on costs of major surgical procedures: a cost analysis of 1200 patients. Ann Surg 2011;254:907-13.

8 National health Service (NHS) Digital. Standards and collections. IBS 0070: healthcare resource groups (HRGs). Available: https://digital. nhs.uk/data-and-information/information-standards/informationstandards-and-data-collections-including-extractions/publicationsand-notifications/standards-and-collections/isb-0070-healthcareresource-groups-hrgs [Accessed Feb 2021].

9 National Health Service (NHS) Digital. OPCS classification of interventions and procedures version 4.8, 2017. Available: https:// classbrowser.nhs.uk/\#/book/OPCS-4.8 [Accessed Feb 2021].

10 National Health Service (NHS) Digital. HRG4+ 2017/2018 reference costs grouper. Available: https://digital.nhs.uk/services/nationalcasemix-office/downloads-groupers-and-tools/costing-hrg4-201718-reference-costs-grouper [Accessed Feb 2021].

11 National Health Service (NHS) Improvement. 2017/2018 reference costs and guidance. Available: https://improvement.nhs.uk/ resources/reference-costs/ [Accessed Feb 2021].

12 National Health Service (NHS) Improvement. National tariff 2017/19: documents and policies. Available: https://improvement.nhs.uk/ resources/developing-the-national-tariff/\#past [Accessed Feb 2021].

13 National Health Service (NHS) Digital. Hospital episode statistics (HES). 2017-18. Available: https://digital.nhs.uk/data-andinformation/data-tools-and-services/data-services/hospital-episodestatistics [Accessed Feb 2021].

14 Getting it right first time (GIRFT). Available: https://www.gettingitrig htfirsttime.co.uk/surgical-specialty/general-surgery/ [Accessed Feb 2021].

15 National Institute for Health and Care Excellence (NICE). Surgical site infections: prevention and treatment. NICE guideline [NG125]. Health economic model report. 11 April 209. Available: https://www.nice. org.uk/guidance/ng125/documents/economic-report [Accessed Feb 2021].

16 News: long NHS waiting times are harming patients, MPs warn. BMJ 2019;365.

17 Habib K, Daniels S, Lee M, et al. Cost implications and oncological outcomes for laparoscopic versus open surgery for right hemicolectomy. Annals 2016;98:212-5.

18 Bosanquet DC, Ansell J, Abdelrahman T, et al. Systematic review and meta-regression of factors affecting midline incisional hernia rates: analysis of 14618 patients. PLOS One 2015;10:e0138745.

19 Capita. The quality of clinical coding in the NHS. Payment by results data assurance framework. September 2014. Available: https://www. chks.co.uk/userfiles/files/The_quality_of_clinical_coding_in_the_ NHS.pdf [Accessed Feb 2021]. 